\title{
Depth-Resolved XMCD Study of Ultrathin Mo/Co/Au Films
}

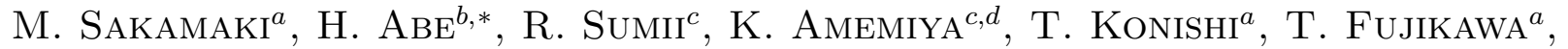 \\ L.T. Baczewski ${ }^{e}$, A. Wawro ${ }^{e}$, A. Petroutchik ${ }^{e}$, Z. Kurant ${ }^{e}$ and A. Maziewski ${ }^{f}$ \\ ${ }^{a}$ Graduate School of Advanced Integration Science, Chiba University \\ Yayoi-cho 1-33, Inage, Chiba 263-8522, Japan \\ ${ }^{b}$ Graduate School of Science, The University of Tokyo \\ 7-3-1 Hongo, Bunkyo-ku, Tokyo 113-0033, Japan
}

${ }^{c}$ Institute of Materials Structure Science, High Energy Accelerator Reseach Organization

1-1 Oho, Tsukuba-shi, Ibaraki 305-0801, Japan

${ }^{d}$ Japan Science and Technology Agency, CREST

${ }^{e}$ Institute of Physics, Polish Academy of Sciences

al. Lotników 32/46, 02-668 Warsaw, Poland

${ }^{f}$ Institute of Experimental Physics, University of Białystok

Lipowa 41, 15-424 Białystok, Poland

\begin{abstract}
The effect of Mo overlayer on the magnetism of ultrathin Co films grown on $\mathrm{Au}(111)$ was studied. The structure and magnetism at the Mo/Co interface were studied by means of depth-resolved X-ray magnetic circular dichroism. The magnetic moment of Co near the Mo/Co interface was reduced in comparison to the inner part of the film for the samples which have in-plane anisotropy. This effect is more prominent in the case of Mo overlayer with higher roughness, and can be attributed to Co-Mo intermixing at the interface.
\end{abstract}

PACS numbers: 75.30.Gw, 75.70.Cn, 78.70.Dm

\section{Introduction}

Ultrathin magnetic films have many interesting magnetic properties that are different from bulk systems. Understanding and modification of these properties are of great importance both for fundamental science and technological applications such as magnetic/magneto-optical recording media. One of the important aspects of these systems is the possibility of controlling magnetic properties such as magnetic anisotropy by adding different kinds of overlayers [1-4]. Magneto-optical analysis shows that $\approx 1 \mathrm{~nm}$ silver overlayers induce the spin reorientation transition (SRT) between in-plane and out-of-plane magnetization [1]. Significantly smaller thickness of Mo or V is sufficient to tune the SRT [3]. It is crucial to study the magnetism and structure of the interface in order to understand the role of different overlayers. In general, characterization of buried interfaces is not easy to per-

\footnotetext{
* Present address: Department of Chemistry, Faculty of Science and Technology, Keio University, 3-14-1 Hiyoshi, Yokohama, 223-8522, Japan
}

form and requires special methods. Recently, Postava et al. [2] showed decrease in magnetic moment in two atomic layers of the Co film in the vicinity of $\mathrm{V} / \mathrm{Co}$ and Mo/Co interface of $\mathrm{V}$ and Mo covered ultrathin films, by fitting the ellipticity and polar Kerr rotation simultaneously acquired in magneto-optical measurements. Alternatively, here we applied depth-resolved soft X-ray spectroscopies on Mo/Co films in order to obtain more direct information about magnetic and electronic structure of the interface.

Ultrathin $\mathrm{Mo} / \mathrm{Co} / \mathrm{Au}$ shows a rich variety of magnetic anisotropy, including in-plane/out-of-plane SRT and change in the in-plane anisotropy depending on the thickness of Co film and Mo overlayer, as confirmed by magneto-optical Kerr effect (MOKE) measurements [3]. In the following, we show the results of depth-resolved soft X-ray magnetic circular dichroism (XMCD) measurements, which are capable to obtain the depth profile of the magnetic moment of Co atoms. These results are correlated with the information from MOKE measurements which probe the whole film. 


\section{Experimental}

Samples were grown in a molecular beam epitaxy (MBE) system operating in the low range of $10^{-10}$ Torr vacuum equipped with effusion cells and electron guns. Growth process was monitored in situ by reflection high energy electron diffraction (RHEED) and Auger electron spectroscopy (AES). A $10 \mathrm{~mm} \times 10 \mathrm{~mm}$ polished sapphire $\mathrm{Al}_{2} \mathrm{O}_{3}$ (11-20) single crystal wafers were used as substrates. The $20 \mathrm{~nm}$ bcc $\mathrm{Mo}(110)$ buffer layer was grown at $1000^{\circ} \mathrm{C}$. The $20 \mathrm{~nm}$ fcc $\mathrm{Au}(111)$ layer was deposited at room temperature onto the Mo buffer. Then we deposited $2.5 \mathrm{~nm} \mathrm{hcp} \mathrm{Co}(0001)$ and wedged-shaped Mo overlayer in order. The whole sample was finally capped with $2 \mathrm{~nm}$ of Au coverage. The thickness of the Mo layer wedge varied from 0 to $1.0 \mathrm{~nm}$. Figure 1 shows schematic illustration of the grown structure. We obtained different roughness of the Mo overlayer by controlling the deposition temperature. Two samples were prepared, one of them has epitaxially grown Mo overlayer and the other has polycrystalline $\mathrm{Mo}(110)$ overlayer. The quality of the interfaces and the crystallographic orientation were monitored in situ by RHEED.

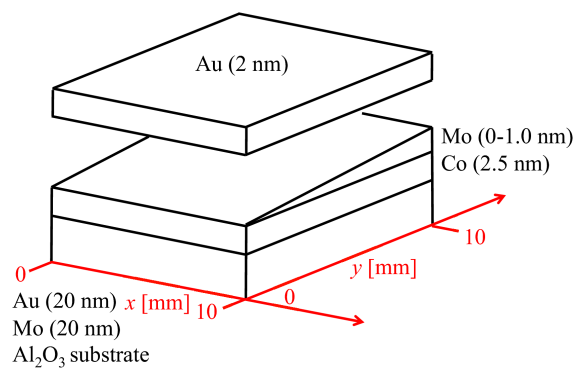

Fig. 1. Schematic illustration of $\mathrm{Co} / \mathrm{Au} / \mathrm{Mo} /$ $\mathrm{Al}_{2} \mathrm{O}_{3}(11-20)$ thin films with wedged Mo overlayer. Mo thickness varies along $y$-axis.

We performed MOKE measurements for both samples, and found that they have in-plane magnetic anisotropy in the whole area of the samples, in agreement with the earlier study [3]. We obtained square longitudinal MOKE hysteresis loops when the magnetic field was applied along the sample plane.

The depth-resolved XMCD developed by Amemiya et al. $[5,6]$ provides depth-resolved and element specific magnetic information. Experimental setup is schematically shown in Fig. 2.

The emitted electrons were collected separately at different detection angles $\left(\theta_{\mathrm{d}}\right)$ by using an imaging type detector consisting of a micro-channel plate (MCP), a phosphor screen and a CCD camera. All the spectra with different probing depths were recorded at once. Partial electron yield mode with a retarding voltage of $500 \mathrm{~V}$ was adopted, so that the Co $L M M$ Auger electrons were mainly collected. XMCD measurements were performed on the samples remanently magnetized by pulse magnetic field $(\approx 700$ Oe) before each measurement carried out

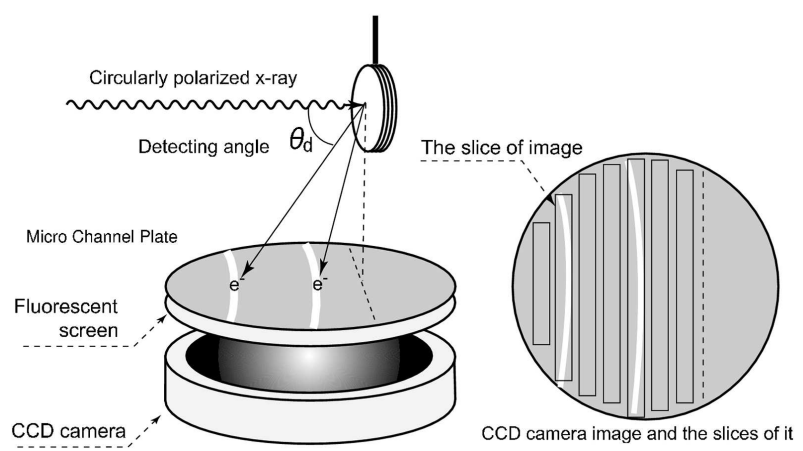

Fig. 2. Layout of the experimental setup of the depth-resolved technique. We obtain spectra with different effective electron escape depth at each slice. Grazing $\mathrm{X}$-ray incidence angle is used when we probe in-plane magnetization (see text).

at room temperature. All the measurements were performed in a vacuum chamber at beamlines BL-7A and $11 \mathrm{~A}$ at the Photon Factory in High Energy Accelerator Research Organization, Japan. The degree of circular polarization $\left(P_{\mathrm{c}}\right)$ was $\approx 0.8$.

\section{Result and discussion}

Figure 3 shows Co $L$-edge XMCD spectra at $y=2 \mathrm{~mm}$, and $y=9 \mathrm{~mm}$ where the thickness of Mo overlayer is equal to $\approx 0.2 \mathrm{~nm}$ and $\approx 0.9 \mathrm{~nm}$, respectively. All the spectra in Fig. 3 were measured at grazing X-ray incidence $\left(60^{\circ}\right.$ from normal $)$, that corresponds to the projection of the in-plane magnetic component in that direction. The spectra have been normalized by the $L_{2,3}$ edge jump, so that the XMCD intensity is related to the magnetic moment per Co atom. Detailed analysis including the precise determination of the probing depths and atomic layer resolved spectra will be reported elsewhere. Here, in order to estimate roughly the depth profile, we divided the electron yield signal into three parts: (1) spectra taken at small detection angles, which are most sensitive to the Mo/Co interface region, (2) spectra taken at larger detection angles, which contain more contribution from deeper parts of the Co layer and (3) total electron yield spectra, where the electron escape depth is much larger than that of the partial electron mode, so that the whole Co layer is rather equally contributing to the spectra. We definitely see the decrease in the XMCD intensity near the $\mathrm{Mo} / \mathrm{Co}$ interface compared to the inner part of the Co layer for both samples. This effect is more pronounced in the sample with polycrystalline Mo overlayer. These results indicate that magnetic moments of Co atoms near the Mo/Co interface are drastically reduced by the Mo overlayer. The stronger overlayer effect in case of the rougher interface may be explained as a result of larger degree of intermixing in that case. Also, we expect that charge transfer and oxygen transport between Co and Mo, if any, may substantially affect the magnetism at the interface. Further discussion of these 


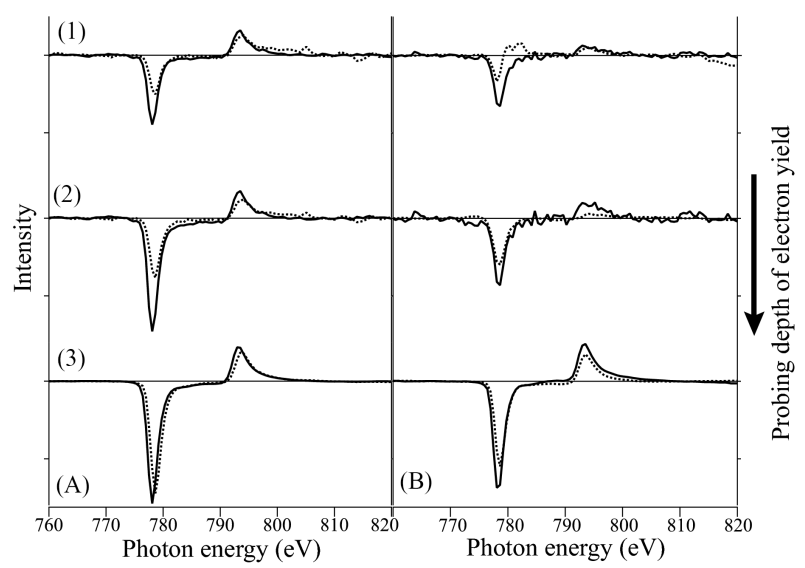

Fig. 3. Co $L$-edge XMCD spectra at (A) $y=2 \mathrm{~mm}$ and (B) $y=9 \mathrm{~mm}$ with varying probing depth. Curve (1) has the shortest effective electron escape depth and therefore is sensitive to Mo/Co interface, where the slices which are the nearest to the samples (i.e. the smallest detection angles) have been added. Curve (2) has larger probing depth, so that it contains more information on the middle part of Co layer, where the further slices were added. Curve (3) was measured in total electron yield mode, in which much larger contribution from inner part of Co layers is included compared to the partial electron mode. Solid curves and broken curves correspond to the sample with epitaxial Mo overlayer and polycrystalline Mo overlayer, respectively. Decrease in the XMCD intensity was observed near the interface.

effects using X-ray photoemission spectroscopy (XPS) and results of extended X-ray absorption fine structure (EXAFS) will be published elsewhere, where structural information such as disorder and intermixing of $\mathrm{Co}$ and Mo atoms will be shown.

\section{Conclusion}

We obtained the depth profile of the magnetic moment of Mo covered Co ultrathin films by means of depth-resolved XMCD technique. In agreement with the MOKE measurements, XMCD results showed in-plane magnetization in the whole area of both samples. We found that magnetic moment of Co atoms near the Mo/Co interface was drastically decreased. This effect is more prominent in the case of Mo overlayer with higher roughness.

\section{Acknowledgments}

The authors would like to thank Dr. E. Holub-Krappe and Dr. D. Arvanitis for helpful and stimulating discussions. This work was supported by Marie Curie Fellowship for "Transfer of Knowledge" (NANOMAG-LAB, No. 2004-003177) and by the Polish National Scientific Network ARTMAG "Magnetic nanostructures for spintronics".

The XMCD measurements were performed under the approval of the Photon Factory Program Advisory Committee (PF-PAC No. 2006G227).

\section{References}

[1] M. Kisielewski, A. Maziewski, M. Tekielak, A. Wawro, L.T. Baczewski, Phys. Rev. Lett. 89, 087203 (2002).

[2] K. Postava, Z. Kurant, A. Maziewski, A. Stupakiewicz, L.T. Baczewski, A. Wawro, M. Aoyama, T. Yamaguchi, Appl. Surf. Sci. 254, 360 (2007).

[3] Z. Kurant, R. Gieniusz, A. Maziewski, M. Tekielak, W. Stefanowicz, I. Sveklo, V. Zablotskii, A. Petroutchik, L.T. Baczewski, A. Wawro, J. Magn. Magn. Mater. 316, e511 (2007).

[4] Y. Huttel, G. van der Laan, T.K. Johal, N.D. Telling, P. Bencok, Phys. Rev. B 68, 174405 (2003).

[5] K. Amemiya, S. Kitagawa, D. Matsumura, H. Abe, T. Ohta, T. Yokoyama, Appl. Phys. Lett. 84, 936 (2004).

[6] K. Amemiya, S. Kitagawa, D. Matsumura, T. Yokoyama, T. Ohta, J. Phys., Condens. Matter 15, S561 (2003). 